\title{
A Real-Time Monitoring System of Energy Measurement
}

\author{
Aiguo $\mathrm{Li}^{1, \mathrm{a}}$, Jing $\mathrm{He}^{2, \mathrm{~b}}$, Jiaojiao $\mathrm{Du}^{3, \mathrm{c}}$, Qi Yang ${ }^{4, \mathrm{~d}}$,Wenkai Wang ${ }^{5, \mathrm{e}}$ \\ 1,2,3,4,5 School of Computer science and Technology, \\ Xi'an University of Science and Technology \\ Xi'an, 710054,China \\ ali_ag@sina.com, ${ }^{\mathrm{a}}$ hejing_1230@163.com, 'dujiao8675@126.com, \\ dsniper-yang3@sohu.com, e670405621@qq.com
}

Keywords: Energy Measurement, Real-Time Monitoring, B/S Structure

\begin{abstract}
Real-time monitoring of energy measurement is a crucial and challenging field of application research. Development of practical real-time monitoring system of energy measurement has important practical significance. Measurement of a plant's energy requirements are analyzed, The real-time monitoring of energy measurement are based on B / S structure design, and the actual problems are analyzed and discussed in the real-time monitoring system. The system's design and implementation method are analyzed in the practical application, to meet the requirements of real-time system. the real-time data collection, data analysis, data flow management are involved.
\end{abstract}

\section{Introduction}

Highly efficiency measurement of enterprise energy management can bring huge economic benefits for the enterprise. Petrochemical, metallurgy, electric power and other industries with higher energy consumption has always attached great importance to the measurement of enterprise energy, energy measurements management of these enterprises system was built completely in China. Various losses and waste are inevitably emerged in all aspects of other industry companies from production to consumption ${ }^{[1]}$.

Being a major method of energy measurement, energy measurement is thought to have huge impact on enterprises, such as economic benefits and become more competitive ${ }^{[2]}$. So establishing a relatively complete real-time monitoring system can play an important role to ensure the measurement the energy companies measurement go well. Being a management tool and method at the same time, using the energy measure and control real-time data collection, diagnosis, analysis, implementation of effective management, scientific and accurate measurement data could guide energy using, so as to achieve the purpose ${ }^{[3]}$.

The study is focus on key technology of the energy management based on B / S structure. Thus research is based on B / S structure of the energy management system and lay a solid foundation. In this paper, the energy management based on B / S structure of is dicussed in some of the research focus. Finally, these techniques will be used in real-time monitoring system of energy measurement. 


\section{The System Architecture of Real-time Monitoring System of Energy Measurement}

\subsection{System Requirements}

\subsubsection{System Functional Requirements}

Real-time data monitoring system for energy measurement mainly includes the following functional requirements: the monitor screen function, the data monitoring queries function, the monitoring statistical analysis function, the running the report function, the logging alarm function, the real-time database function, the collection spreading point about data function, data transmission network traffic Estimated consumption functions, and the user management function.

2.1.2 System Performance Requirements

1) Openness: With the users and developers' interactive cooperation, the system should be more open. As time goes by, each module would be more perfect, also become a scalable system

2) Flexibility: According to business needs, the work processes can customize, and the system design is flexibility for customers, which could help businesses to complete their daily work easily day by day.

3) Data Sharing: The system has a powerful query statistical functions, information can be queried by any combination of query conditions, data also can be analyzed in all kinds of information graphics vividly, excel and other report formats can be generated.

\subsection{The Level of System Designing}

Real-time monitoring system is mainly composed of five levels as illustrated below Figure 1:

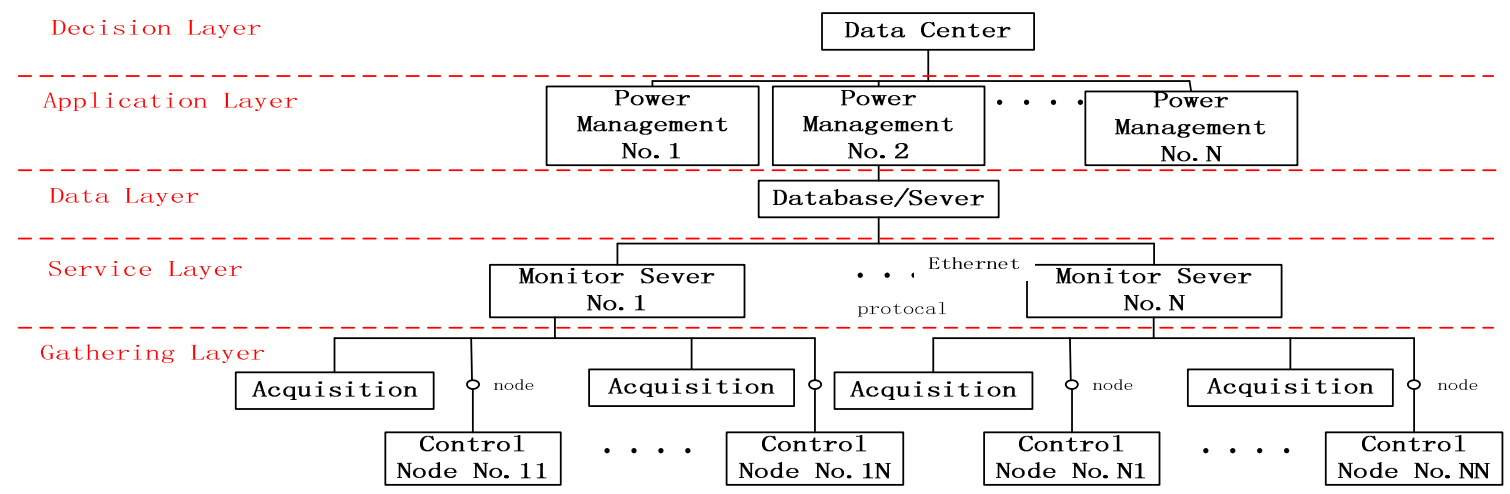

Figure 1 System-level of Data structure

Real-time monitoring system of energy measurement data is divided into five layers, including the gathering layer, service layer, data layer, application layer and decision layer.

(1) Gathering layer: equipments are used to control the collected data node of real-time data acquisition in gathering layer, datas are sent form the service layer to the gathering layer according to certain communication protocol.

(2) Service layer: datas are transmitted from the control layer which collecte all the dates to data layer throughing ethernet.

(3) Data Layer: real-time data is stored from the data services layer to database ,to facilitate the application layer. 
(4) Application layer: Application layer through the query functions, statistical functions or a real-time data reporting will be displayed in many ways.

(5) Decision-making layer: a variety of data generated or static curve of the dynamic curve through the application layer to facilitate management of decision-making are provided.

\subsection{Real-time Monitoring of Enterprise Energy Measurement System Operating Environment}

The Microsoft Corporation. NET technology framework are used in the software, and the main software requirements are as follows:

Application Server: Windows 2000 Server or Windows 2003 Server operating system.

Application database server above II6.0: SQL Server2005.

\section{Energy Measurement System Features Real-time Monitoring}

According to the system's functional requirements, the energy measurement data monitoring module consists of seven modules. The figure 2 shows the system's structure.

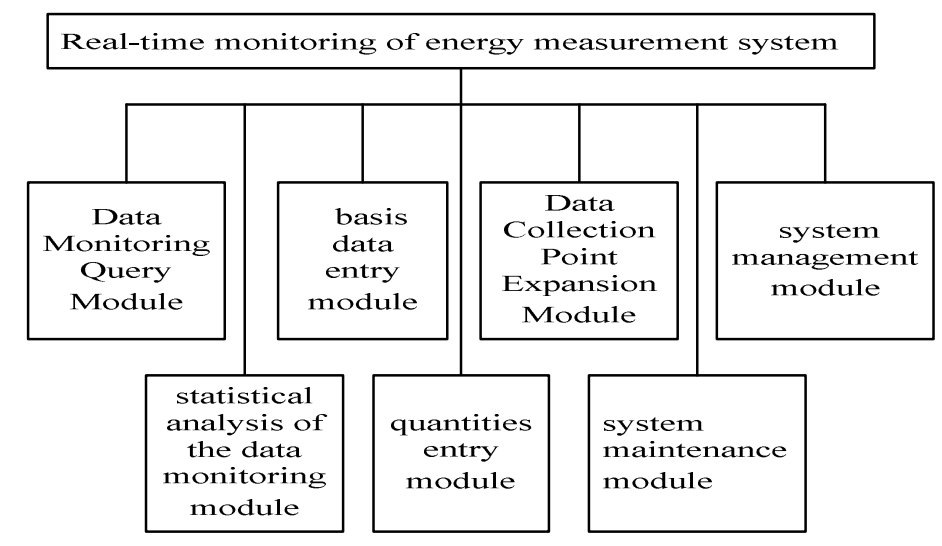

Figure 2 System Function Structure

\section{1) Data Monitoring Query Module}

The main function of monitoring query data is monitoring all data for enterprise. History data stored in the database can be queried from all historical data, the query time interval and time range specified by the user, the query interval methods include: minutes, hours, days, weekly, monthly, quarterly and annual.

\section{2) Statistical Analysis of Data Monitoring Module}

Statistical analysis of data for monitoring can statistical the unit of time period consumed information and other information in a variety of techniques, can be consumed within a certain period of time, the total power to curve or histogram could be generated, to facilitate customers' view or decision-making.

\section{3) Data Collection Point Expansion Module}

The system can not only monitor the data of collection points, but also support extensions of data collection points, When the collection points are increased or decreased, the system can still ensure the accuracy and completeness of data monitoring. 


\section{4) System Maintenance}

The data tree structure is used to display energy measurement data of real-time monitoring in system maintenance, and it divided by different regions, each region is regarded as a single unit, on-site measurement of the value of the collection point is real-time displayed. The image feature is the ability to manage all collection points, the picture is simple and can directly compare the various parameters, for easy viewing of all parameters are abnormal. The function mainly shows the cumulative values and the instantaneous values of the collection points, and makes fault alarm for this specific situation.

\section{5) System Administration}

User management module is divided into three roles : super administrators, administrators and ordinary users. Super Administrators can manage all real-time monitoring data and information in enterprise, can set about other user's permissions. Administrators have specific query permissions about statistical information management and information for the entire software system ,they protect the security and reliable operation of the system. Ordinary users can query datas for requiring.

6) Basis Information Management

According to the different user's role,monitoring information is managed to assign administrative rights on the basis of the information. Basis information on enterprises to ensure security in the unified management of the premise.

7) Quantity Input

All production information of enterprises are managed in the quantity input module, the management authority is allocated, for using production information in the data analysis according to the different role.

\section{Key Technologies}

Based on the development and trends of the existing energy monitoring and management, the key technologies based on B / S structure for energy management are focused on the three aspects as follows:

One. Information Collection: A more humanity method of reading the table is used, without manual operation, synchronization service program is developed by. NET Framework, this service is deployed to the enterprise server, the synchronization service program is in accordance, read data and preliminary data processing with the requirements specified intervals time, which makes energy information obtain the required business data related to Energy measurement automatically, and without manual operation of the collection to the database server.

Two: Information Analysis: The traditional method of manual analysis of data is tedious and error-prone, and real-time data management system for energy measurement can be obtained in the data collection of useful data for intelligence analysis, minimizing manual operations to save costs. according to the demand for relatively complex operations or treatment. The real-time monitoring 
system of energy measurement information will be collected data and analyzed on demand. And this system also can be self-learning, self-improvement, better for the next hub of support for policy-makers to provide intelligent analysis. Dynamically adjust the load to be the amount of business to reduce energy costs, a reasonable allocation and regulation of energy use across the enterprise, could improve economic efficiency of enterprises.

Three: Information Released:

1) The manual delivery of the report can not be well represented in the form of real-time system, it can not be display data instantaneous visual, the system can release the information needs of enterprises needs to know if the release of the data and information, and display out intuitive, to facilitate business decisions. System, large amounts of data can be exported directly from the client in the user save the Excel permanent preservation and use.

2) After collection data is intelligent analyzed, although the form of statements derived fast, easy to operate, but it can not display the intuitive use of energy, it can not reflect the business trends in energy use. The energy measurement data monitoring system in order to overcome this drawback, use dynamic or static graphics are used to visualize the response of the energy situation, not only beautiful, but also user-friendly graphical seen from the problems and solutions, this system can improve the energy use rate, thus save the cost of business and energy conservation.

\section{Conclusion}

The enterprise real-time monitoring system of energy measurement based on B / S structure has been achieved, and it has been tested in the XX factory for two months, laying 32 monitoring points overall, almost millions of data are monitored, the system's various functionals was running well in the process of trial operation.

(1) The system is monitoring production line of electrical energy of the factory XX, responding to the operation of the various monitoring points accurate and timely, and displaying with screen in the system monitoring intuitive, the enterprises distribute energy dynamically, which is based on the data of monitoring points, improve energy efficiency, so that energy conservation, cost saving.

(2) Energy measurement monitoring system not only monitors the energy of factory, but also completes the XX factory energy information management. The system avoids the cumbersome manual operation and ease of bringing the wrong, and improves the work efficiency of employees and the enterprise create more value eventually.

\section{References}

[1] WenyingWu. Energy monitoring measurement for of steel plant. Energy Enginneering[J].2000,6

[2] Aniket A.Shah. Energy Measurement Technique for Computing System.Patent Application Publication[J].Dec31,2009 sheet 6 of 6.

[3] Mahmood Ali,Sai Kumar Ravula. REAL-TIME SUPPORT AND ENERGY EFFICIENCY IN WIRELESS SENSOR NETWORKS - Technical report[J].IDE0805,January 2008 [J]. 